This is the peer reviewed version of the following article:

Rodriguez-Moreno, S., Farchione, T. J., Roca, P., Marín, C., Guillén, A. I., \& Panadero, S. (2020). Initial effectiveness evaluation of the Unified Protocol for Transdiagnostic Treatment of Emotional Disorders for homeless women. Behavior Modification, 1-23,

which has been published in final form at:

https://doi.org/10.1177/0145445520982562

This article may be used for non-commercial purposes in accordance with SAGE Journals and Conditions for Use of Self-Archived Versions. 
Accepted version of the article:

Rodriguez-Moreno, S., Farchione, T. J., Roca, P., Marín, C., Guillén, A. I., \& Panadero, S. (2020). Initial effectiveness evaluation of the Unified Protocol for Transdiagnostic Treatment of Emotional Disorders for homeless women. Behavior Modification, 1-23. DOI: doi.org/10.1177/0145445520982562

Copyright: SAGE Journals.

\title{
INITIAL EFFECTIVENESS EVALUATION OF THE UNIFIED PROTOCOL FOR TRANSDIAGNOSTIC TREATMENT OF EMOTIONAL DISORDERS FOR HOMELESS WOMEN
}

\author{
Sara Rodriguez-Moreno ${ }^{1}$, Todd J. Farchione ${ }^{2}$, Pablo Roca ${ }^{1}$, Carolina Marín ${ }^{1}$, Ana
} Isabel Guillén ${ }^{1}$ and Sonia Panadero ${ }^{1}$

1. Clinical Psychology Department, School of Psychology, Complutense University of Madrid, 28223, Madrid, Spain.

2. Center for Anxiety and Related Disorders, Department of Psychology, Boston University, 648 Beacon Street, Boston, MA 02215, USA.

Corresponding author: Sara Rodriguez-Moreno, Clinical Psychology Department, Psychology School, Complutense University of Madrid, 28223, Madrid, Spain. Email: sarairod@ucm.es

Acknowledgments: Sara Rodriguez-Moreno is a $\mathrm{PhD}$ candidate at Complutense University of Madrid and this work will be part of her PhD work on mental health and psychological treatments in homeless women. The authors want to thank all women experiencing homelessness for their generosity to participate in the study. We want to thank San Isidro Center, La Rosa Center, Puerta Abierta Center, Luis Vives Center, Geranios Center and Council of Madrid for the immense help of the recruitment of the participants and the availability of their workers and rooms to carry out the program.

Funding Source Declaration: This work was supported "Agencia Estatal de Investigación" of the "Ministerio de Economía y Competitividad" of Spain [grant number FEM2016-75317-R]; and the Complutense-Santander Back [grant number CT17/17CT18/17] to SRM.

Declaration of Interest: Dr Farchione reported receiving royalties from Oxford University Press as one of the authors of the Unified Protocol. He also receives research support from the National Institute of Health. No other disclosures were reported. 


\begin{abstract}
The purpose of this study is to evaluate the effectiveness of the Unified Protocol for Transdiagnostic Treatment of Emotional Disorders adapted for homeless women (UPHW). Eighty-one homeless women participated in this single-blinded quasiexperimental clinical trial, involving up to 12 sessions of group treatment, and 3-and 6month follow-ups. The participants received either immediate treatment with the UPHW $(n=46)$ or delayed treatment, following a 12-week wait-list control period (WLC; $n=$ 35). Primary outcomes included depression and anxiety. Secondary measures comprised positive, and negative affect, psychological well-being, health perception, and social support. The UPHW resulted in significant improvement on measures of anxiety, depression and negative affect. Improvements in anxiety and depression were maintained over a 3-month follow-up period, but not at 6-month. The reliability of the clinical changes showed significant differences between UPHW and WLC for depression. Moreover, the inter-session assessment in the UPHW group showed a linear trend reduction for depression and anxiety scores along the 12 sessions. The clinical implications on the UPHW in social settings are also discussed.
\end{abstract}

Keywords: homelessness, homeless women, mental health, transdiagnostic treatment, unified protocol. 


\section{Introduction}

Homelessness is characterized by extreme poverty and social exclusion in high income countries. It is a significant social issue that affect millions of people worldwide each year (Fransham \& Dorling, 2018). The economic development in high-income countries is in sharp contrast with the exponential growth of homelessness that has occurred worldwide during the last three decades: around four million people are in a homeless situation each year in Europe and around three million in the USA (Fazel et al., 2014). In the case of Spain (country where the study was conducted), according to the National Institute of Statistics (2018), 18,001 people were housed on a daily basis in homeless care centers.

Finding a definition of homelessness is not a straightforward task. Most homelessness definitions have been coined in specific contexts and countries, and multiple definitions have been proposed. The European Typology of Homelessness and Housing Exclusion (ETHOS) define that a homeless person can be found in the following categories (Amore et al., 2011): (1) rooflessness (people sleeping rough, in a night shelter), (2) houselessness (shelters and supported accommodation for formerly homeless people), (3) insecure housing (people living under threat of violence and move temporarily with family/friends), and (4) inadequate housing (living in extreme overcrowded conditions, unfit housing). Furthermore, attention should be paid to the "hidden homeless" phenomenon (Mayock \& Sheridan, 2012; Watson et al., 2016), referred to those people who lose their apartments or houses, and instead of going directly to a shelter or the street, they may live temporarily with their family, friends or sleep in rooming houses.

Research to date consistently demonstrates that mental health problems are a key factor of homelessness. A two-way relationship between mental health and homelessness has been found (Duke \& Searby, 2019), where mental health problems act as a vulnerability factor and as a consequence of homelessness (Chambers et al., 2014). First, some studies suggest that mental health problems are one of the main risk factors for becoming and remaining homeless (Nilsson et al., 2019). For example, it has been found that people with mental health problems are twice as likely to experience homelessness at some point in their lives as people without mental health problems (Australian Bureau of Stadistics, 2016), probably because mental health problems can lead to significant impairments in functioning that can contribute to homelessness (Llerena et al., 2018). Second, homeless people suffer a higher prevalence of mental health problems than the general population (Fazel et al., 2008), which may be even greater when considering the high prevalence of stressful life events and its effects on mental health (Lund et al., 2010). Meta-analytic evidence suggests that the pooled prevalence rate of mental disorders in homeless population is $77.5 \%$ (Schreiter et al., 2017). According to the geographical location, the prevalence of mental health problems in homeless people the USA is $57.8 \%$ (U.S. Department of Housing and Urban Development, 2015), in contrast to the $18.9 \%$ in the general population (National Institute of Mental Health, 2017). Data in Spain suggest that the prevalence of mental health problems among homeless people ranges from 25 to $35 \%$ (Vázquez \& Muñoz, 2001). The discrepancy and variability in the prevalence of mental health problems between different studies may be related to methodological differences between studies, such as the type of homeless condition being evaluated (e.g., sleeping on the streets, using shelters, etc), the inclusion criterion in the definition of mental disorder (e.g., including or not substance abuse), as well as cross-cultural and context differences. 
The most prevalent mental disorders among homeless people are affective disorders, substance and alcohol abuse, psychotic disorders, personality disorders, and post traumatic disorder (Fazel et al., 2008; Hossain et al., 2020). Furthermore, comorbidity rates between different mental health problems are very high in the homeless population (Urbanoski et al., 2018). Homeless people are four times more likely to present more than one psychological disorder at the same time than the general population (National Institute of Mental Health, 2009), possibly indicating greater clinical severity. Although homeless women make up an increasing portion of the homeless population, a growing body of research suggest that homeless women present more mental health problems than their male counterparts and even more than general population women (Muñoz et al., 2005; Welch-Lazoritz et al., 2015). However, research on homelessness have been marked by an androcentric vision (Mayock \& Bretherton, 2016), which has limited our understanding of women in this situation. The difficulties arising from the interaction between the lack of socioeconomic resources and the mental health problems appears to be even more prominent in homeless women (Chambers et al., 2014), which makes them a particularly vulnerable subgroup.

To date, there has been a lack of evidence-based psychological treatments developed to specifically target mental health problems in people experiencing homelessness (Speirs et al., 2013). Most studies have focused on structural factors contributing to homelessness (Hwang \& Burns, 2014) and systemic support interventions providing housing, employment and legal support (Baxter et al., 2019). Although some of these interventions may indirectly improve psychological symptoms, mental health issues are not targeted directly. Speirs and colleagues (2013) conducted a systematic review analyzing existing psychosocial interventions for homeless women. They found that these treatments, which often contained psychoeducation and motivational interviewing, led to reduced distress and alcohol/drug use while improving self-esteem and healthcare use. Furthermore, psychological interventions have also been shown to improve mental health and social outcomes among women in shelters due to intimate partner violence (Lako et al., 2013). Despite this, existing studies show several limitations (such as the lack of comparison groups and small sample size) and further research is needed to ensure the effectiveness of psychological interventions for homeless women (Speirs et al., 2013).

Considering the high prevalence of emotional disorders and comorbidity in this population, transdiagnostic protocols may offer a more effective alternative to provide empirically supported treatment to homeless women. One of the most widespread, empirically supported, transdiagnostic treatments for anxiety and depressive disorders is the Unified Protocol for Transdiagnostic Treatment of Emotional Disorders (UP; Barlow et al., 2011). The UP is a manual-based cognitive behavioral therapy focused on psychopathological mechanisms contributing to the development and maintenance of different emotional disorders, namely, frequent and intense negative emotions (i.e., neuroticism), negative reactions to these emotions, and a tendency to avoid or suppress them (Barlow et al., 2014), instead of focusing on each single disorder separately. This allows the application of the protocol to a variety of disorders simultaneously, hence reducing the comorbidity among disorders. The UP has demonstrated large, and stable, symptom reductions across measures of depression, generalized anxiety, panic disorder, social anxiety, obsessive compulsive disorder, post-traumatic stress disorder, and borderline personality across different internalizing disorders (Sakiris \& Berle, 2019). To date, two initial studies have examined the UP for homeless persons, one examining the barriers and facilitators (Youn et al., 2019), and the other evaluating the acceptability and 
feasibility (Sauer-Zavala et al., 2019). On the one hand, Youn et al. (2019) found that some of the main barriers when adapting the UP to the homeless population, were the engagement with the intervention, attendance problems, some important needs not addressed by the protocol and lack of resources to apply. Two important facilitators were the perception of utility and all the contents addressed by the program. On the other hand, Sauer-Zavala et al. (2019) found mixed results in acceptability and feasibility for both the patients and the therapist. However, neither of these studies provide data on the effectiveness of the UP adapted to the homeless population.

Therefore, the primary aim of this study is to evaluate the effectiveness of a group format adaptation of the Unified Protocol for Transdiagnostic Treatment of Emotional Disorders for homeless women. The UP was originally designed for individual application although it has been successfully delivered in groups (Bullis et al., 2015; Osma et al., 2018; Reinholt et al., 2017). Using the UP in this way offers the potential to efficiently treat patients with different disorders in a single group (McHugh et al., 2009). This seems to fit especially well with the structure and needs of community health centers and shelters for the homeless population and has the potential to reduce the burden associated with providing care in these settings. In this study, homeless women were provided the adapted UP (herein referred to as the UPHW) across four different homeless shelters. Primary outcomes included depression and anxiety, whereas secondary measures comprised positive and negative affect, psychological well-being, health perception and social support. We hypothesized that the UPHW would be effective in reducing anxiety and depression symptoms, decreasing negative affect and increasing psychological well-being, positive affect and perceived health of homeless women. This study is expected to contribute to the development of a practical and potentially effective solution for addressing common mental health problems, such as anxiety and depression, in community settings that serve homeless women.

\section{Method}

\section{Study design}

All procedures were approved by the University Ethics Committee of Complutense of Madrid (Ref. 2017/18-004) and the trial was registered at clinicalstrials.gov as NCT04392856. The study was conducted across four centers in Madrid and surroundings areas. The study was originally planned as a randomized controlled trial and was presented to study participants as such; however, the number of homeless women in some shelters was insufficient to permit adequate randomization. Therefore, randomization 1:1 was conducted only in those shelters were the number of homeless women was large enough to generate two groups. Thus, in the end, the study was conducted as a single-blinded quasi-experimental clinical trial.

The design included two conditions: The Unified Protocol experimental group (UPHW) and a Waitlist control condition (WLC). Participants in both conditions, UPHW and waitlist, received psychological and pharmacological Treatment-As-Usual (TAU): women in both groups were allowed to continue to attend the usual shelter services, including individual session with the shelter psychologists, group occupational therapy sessions and employability workshops. Participants assigned to immediate treatment with the UPHW adaptation $(n=46)$ were assessed before and during the intervention (intersession assessment), at the end of treatment, and after a 3 and 6-month follow-up period. Participants assigned to WLC did not immediately receive the intervention for 3 months $(n=35)$, after which they received the same treatment as those in the UPHW 
condition. Participants in the WLC were assessed at the beginning and after the 3-month waitlist period. Following the post-wait-list assessment, these women were assigned to the UPHW treatment and the assessments were then conducted at the end of the intervention and at 3 and 6-month post-intervention. Participation in the study was voluntary and participants did not receive any economic compensation for their participation.

\section{Participants}

A total sample of 81 homeless women from different public shelters in Madrid (Spain) participated in the study. Participants were eligible for the study if they were (1) female gender identity (although all were biologically female); (2) 18 years or older; (3) fluent in Spanish; (4) provided an informed consent; (5) had access to the shelters where the intervention was carried out, (6) able to attend the evaluation and treatment sessions; and (7) to be in the categories 1, 2 or 3 of the ETHOS typology (Amore et al., 2011), contemplated in the proposal made by FEANTSA. Therefore, participants were women in one of the following living situations: (1) women who lived outdoors (on streets, public space or outside); (2) women in emergency accommodation (without habitual place of residence that make night use of shelters); or (3) women in accommodation for homeless people (they live with short to medium intervals in shelters for the homeless, temporary accommodation or temporary supportive accommodation). Furthermore, only women who had attended at least 6 of the 12 sessions were included in the analyses (i.e., minimal treatment exposure), consistent with other studies in this population (Jiga et al., 2019; Santa Maria et al., 2020). Homeless women were excluded if (1) were diagnosed with a severe mental disorder in active phase (i.e. schizophrenia, bipolar disorder or an organic mental disorder); (2) were diagnosed with severe cognitive impairment; or (3) were under the influence of alcohol or other substances determined within the assessment instrument and by the trained interviewers at pre-assessment.

The CONSORT diagram is presented in Figure 1. Eight of 89 homeless women assessed for eligibility were excluded from the trial. Of these, five participants failed to meet inclusion criteria, one woman did not sign the informed consent and two participants declined to participate. Of the 81 homeless women who consented to treatment and participated in the study, $33(71.7 \%)$ completed the post assessment in the UPHW group and $16(45.7 \%)$ in the WLC condition. These dropout rates are similar to those found in previous studies (Coldwell \& Bender, 2007). Sixty-nine homeless women were included in the analysis: 34 in UPHW and 35 in WLC. Participants' mean age was 49.45 $(S D=9.76), 47.8 \%$ were Spaniards, $40.6 \%$ were single, $23.1 \%$ had a high education level and $92.8 \%$ were unemployed. Regarding homelessness variables, the average age of arrival to a homeless situation was 40.45 years $(S D=14.34)$, the average total time in a homeless situation was 7.24 years $(S D=9.10)$ and the average number of times in a homeless situation was 1.83 times $(S D=0.69)$. No significant differences between UPHW and WLC were found in age $\left(t_{(67)}=-0.04, p=.97\right)$, nationality $\left(\chi^{2}(2)=1.22, p=.54\right)$, education $\left(\chi^{2}(5)=3.33, p=.65\right)$, marital status $\left(\chi^{2}(1)=2.01, p=.16\right)$, and employment $\left(\chi^{2}(1)=0.25, p=.62\right)$. Furthermore, no significant differences between groups were found in the age of arrival to a homeless situation $\left(t_{(64)}=0.55, p=.59\right)$, in the total time in a homeless situation $\left(t_{(64)}=0.36, p=.72\right)$, and in the number of times in a homeless situation $\left(t_{(64)}=0.18, p=.86\right)$. Sample size calculation was conducted a priori using G*Power (v. 3.1). With a medium effect size of 0.40 , based on previous studies of the UP in Spain (Osma et al., 2015) and an alpha of .05, it was determined that a total sample size of at least 52 participants would be needed to detect significant effects at $95 \%$ power. 
Figure 1: Study CONSORT diagram.

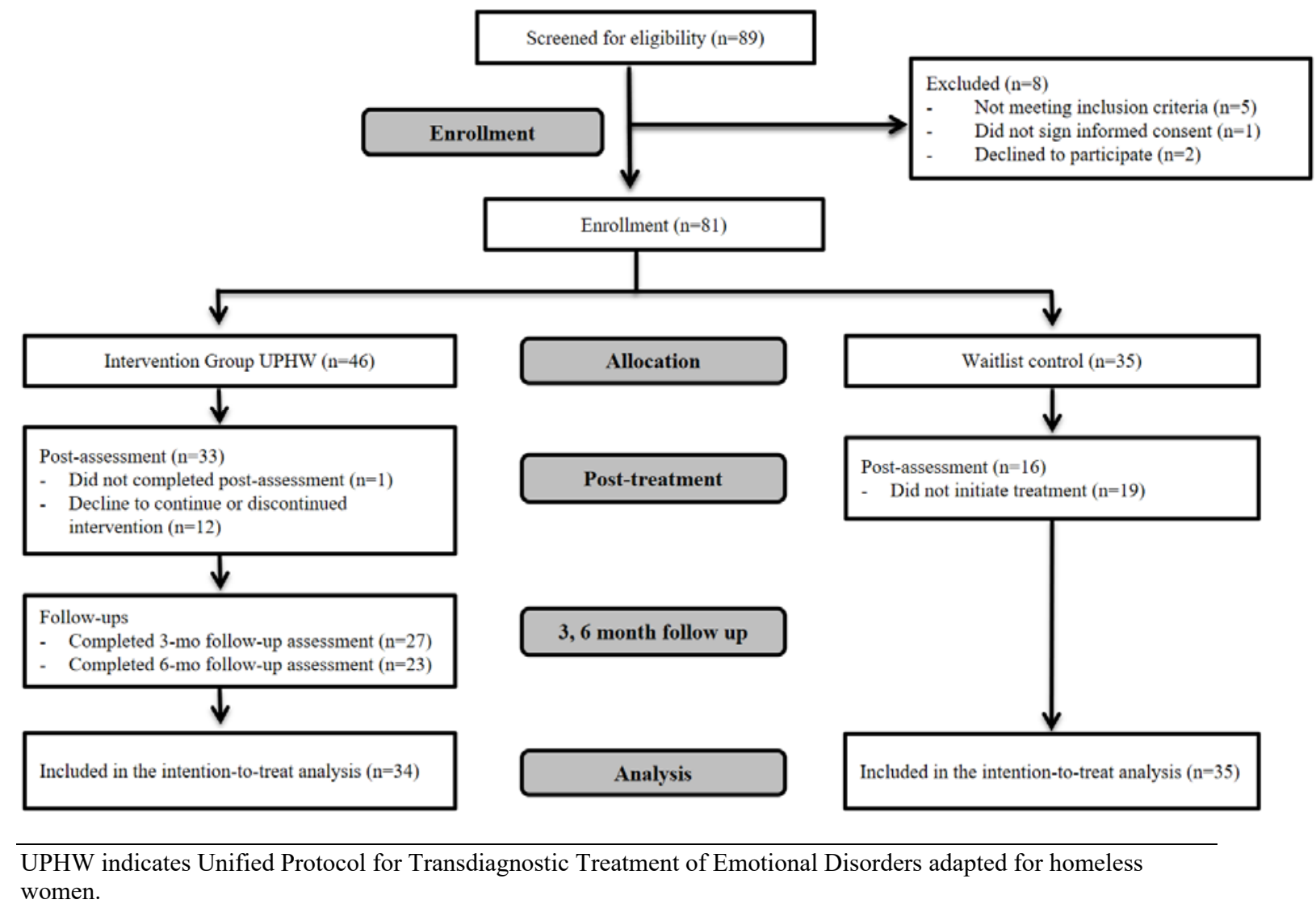

\section{Treatment}

UPHW was an adaptation of the Unified Protocol for Transdiagnostic Treatment of Emotional Disorders (UP; Barlow et al., 2011). Consistent with the original protocol, UPHW consists of five core modules: (a) mindfulness emotion awareness, (b) cognitive flexibility, (c) identifying and preventing patterns of emotion avoidance and maladaptive emotion-driven behaviors, (d) increasing awareness and tolerance of emotion-related physical sensations, and (e) interceptive and situation-based emotion focused exposure. These modules are preceded by an initial module focused on enhancing motivation and an introductory module on the adaptive nature of emotions. Treatment content, by session, is shown in Table 1 in the supplementary material.

In this study, UPHW was delivered in group format (small groups of maximum 10 participants) and consisted of 12 weekly face- to-face sessions lasting 1.5 hours, at a rate of one session per week. In general, some modifications related to the structure, activities and contents of the sessions were carried out to fit homeless women needs living in shelters (Marín et al., unpublished. Available upon request). Information about the therapist and the treatment integrity is provided in the supplementary material.

\section{Assessment}

Study assessments were conducted by independent evaluators who were blind to study condition. Assessments were conducted face-to-face and lasted between 60 and 90 minutes in a private office provided by the shelters. Primary outcome measures were 
severity of anxiety and depression symptoms, as measured by the Beck Anxiety Inventory (BAI) and Beck Depression Inventory-II (BDI-II) respectively. Secondary outcomes measures included: (1) Emotional functioning was measured by assessing the Positive and Negative Affect Scale (PANAS); (2) Integrative well-being was assessed by the Pemberton Happiness Index (PHI); (3) Health status was measured by the Short Form Health Survey (SF-12); (4) Social support was measured by the Social Support Questionnaire (SSQ6); and (5) Anxiety and depression severity and functional impairment, measured with the Overall Anxiety Severity and Impairment Scale (OASIS) and the Overall Depression Severity and Impairment Scale (ODSIS). A detailed description of the instruments is included in the supplementary materials.

\section{Data analysis}

Student $t$ and chi-square test were used to analyze baseline differences between groups. Following CONSORT guidelines (Moher et al., 2012), data imputation was performed following Newman's suggestions (2014), using Maximum Likelihood estimation (ML) via Expectation Maximization imputation (EM). The data analysis plan was conducted with SPSS v. 25 following four successive steps. Firstly, analyses of covariance (ANCOVA) were carried out to examine the pre-post intervention effects, using baseline scores as covariates. Secondly, in order to test whether the postintervention changes remain stable over time, repeated measure ANOVAs were computed for the follow ups (i.e., post intervention, 3-months and 6-months follow up). Thirdly, repeated measure ANOVA was performed to test post-module changes in depression (ODSIS) and anxiety (OASIS) variables. Finally, in order to improve individual-level analysis and the detection of potential adverse effects of the intervention, the Reliable Change Index was computed using the Jacobson and Truax's index (RCI; Jacobson \& Truax, 1991) for the main outcome measures. See supplementary materials for a detailed description of data imputation, the data analysis procedure, and basic assumptions testing.

\section{Results}

\section{Basic assumptions}

The normality assumption was fulfilled only for BDI, PANAS and SF-12 (Kolmogorov-Smirnov $>.05$ ). Homoscedasticity assumption (Levene's test $>.05$ ) and independent assumption (Runs test $>.05$ ) were fulfilled for all the measures. Given that the assumption of normality was only violated for some measures, analyze of variance methods (i.e., ANOVAs and ANCOVAs) remain sufficiently robust and reliable (Schminder et al., 2010).

\section{1) Pre to post intervention effects}

\section{Primary outcome measures (BDI and BAI)}

ANCOVAs analyses on the baseline-corrected post-intervention scores showed a significant group effect for both depression $\left(F_{(1,66)}=16.90, p<.001, \eta^{2}{ }_{\mathrm{p}}=.20 ; 1-\beta=\right.$ $.98)$ and anxiety $\left(F_{(1,66)}=4.79, p=.03, \eta_{\mathrm{p}}^{2}=.07 ; 1-\beta=.58\right)$. Pairwise Bonferroni corrected comparisons indicated that depression and anxiety scores were significantly lower in UPHW than WLC at post intervention (see Table 1). 
ANCOVAs analyses on the baseline-corrected post-intervention scores showed a significant group effect for negative affect $\left(F_{(1,66)}=5.18, p=.03, \eta^{2} \mathrm{p}=.07 ; 1-\beta=.61\right)$. Pairwise Bonferroni corrected comparisons indicated that negative affect was significantly lower in UPHW than in WLC at post intervention (see Table 1). However, ANCOVA analyses did not show significant group effect for positive affect $\left(F_{(1,66)}=\right.$ $.03, p=.88)$, psychological well-being $\left(F_{(1,66)}=1.19, p=.28\right)$, physical health $\left(F_{(1,66)}\right.$ $=.01, p=.92)$, mental health $\left(F_{(1,66)}=.97, p=.33\right)$, general health $\left(F_{(1,66)}=.41, p=\right.$ $.52)$, perceived number of social support $\left(F_{(1,66)}=2.03, p=.16\right)$, and satisfaction with social support $\left(F_{(1,66)}=3.06, p=.09\right)$.

\section{2) Follow up effects of the UP adaptation for homeless women}

Repeated measure ANOVAs were computed to test whether the post changes remain stable over time (i.e., 3-month and 6-month follow up). ANOVAs showed a significant time effect for both depression $\left(F_{(1.57,51.90)}=4.01, p=.033, \eta^{2} \mathrm{p}=.11 ; 1-\beta=\right.$ $.62)$ and anxiety $\left(F_{(2,66)}=10.73, p<.001, \eta^{2} \mathrm{p}=.25 ; 1-\beta=.99\right)$. Pairwise Bonferroni corrected comparisons indicated that depression and anxiety reductions after the intervention remained in the 3-month follow up (post to 3-month $<.05$ ), however, these differences were no longer present at the 6-month follow up (post to 6-month $>.05$ ) (see Table 1). Regarding negative affect, repeated measure ANOVA also showed a significant time effect $\left(F_{(2,66)}=15.60, p<.001, \eta^{2} \mathrm{p}=.32 ; 1-\beta=.99\right)$, however, pairwise Bonferroni corrected comparisons indicated that negative affect reduction after the intervention vanished in the 3-months and 6-months follow up $(p>.05)$. 
Table 1: Presents the means, standard deviations, effect sizes, and coefficient intervals for changes from pre, post and follow ups intervention according to condition ( $\mathrm{n}=69$ )

\begin{tabular}{|c|c|c|c|c|c|c|c|c|c|}
\hline & & $\begin{array}{c}\text { Pre } \\
\text { Mean }(S D)\end{array}$ & $\begin{array}{c}\text { Post } \\
\text { Mean (SD) }\end{array}$ & $\begin{array}{c}\text { Pre-post } \\
\text { Within-group } \\
\text { effect size } d[95 \% \mathrm{CI}]\end{array}$ & $\begin{array}{c}\text { Pre-post } \\
\text { Between-Group } \\
\text { effect size } d[95 \% \mathrm{CI}]\end{array}$ & $\begin{array}{l}\text { 3-month FU } \\
\text { Mean (SD) }\end{array}$ & $\begin{array}{l}\text { 6-month FU } \\
\text { Mean (SD) }\end{array}$ & $\begin{array}{c}\text { Pre-3 FU } \\
\text { Within-group effect } \\
\text { size } d[95 \% \mathrm{CI}] \\
\end{array}$ & $\begin{array}{c}\text { Pre-6 FU } \\
\text { Within-group effect } \\
\text { effect size } d[95 \% \mathrm{CI}]\end{array}$ \\
\hline \multicolumn{10}{|c|}{ Primary outcomes } \\
\hline \multirow{2}{*}{ BDI } & UPHW & $20.82(12.63)$ & $12.83(9.57)$ & $0.62[0.93,0.30]$ & \multirow{2}{*}{$-0.65[-1.14,-0.17]$} & $15.56(11.85)$ & $17.99(12.03)$ & $0.41[0.72,0.10]$ & $0.22[0.57,-0.14]$ \\
\hline & WLC & $18.31(13.87)$ & $20.04(12.14)$ & $-0.12[0.18,-0.42]$ & & NA & NA & NA & NA \\
\hline \multirow{2}{*}{ BAI } & UPHW & $20.97(13.39)$ & $12.72(10.83)$ & $0.60[0.95,0.26]$ & \multirow{2}{*}{$-0.30[-0.78,0.17]$} & $14.80(13.24)$ & $21.11(12.32)$ & $0.45[0.76,0.14]$ & $-0.01[0.25,-0.27]$ \\
\hline & WLC & $18.09(14.07)$ & $15.89(9.72)$ & $0.15[0.45,-0.15]$ & & NA & NA & NA & NA \\
\hline \multicolumn{10}{|c|}{ Secondary outcomes } \\
\hline \multirow{2}{*}{ PA } & UPHW & $29.65(11.62)$ & $30.75(10.18)$ & $-0.09[0.18,-0.37]$ & \multirow{2}{*}{$-0.15[-0.62,0.33]$} & $33.48(13.92)$ & $29.45(11.26)$ & $-0.32[0.10,-0.74]$ & $0.02[0.44,-0.41]$ \\
\hline & WLC & $32.19(10.52)$ & $32.27(10.24)$ & $-0.01[0.38,-0.39]$ & & NA & NA & NA & NA \\
\hline \multirow{2}{*}{ NA } & UPHW & $22.47(9.60)$ & $18.47(9.12)$ & $0.41[0.68,0.13]$ & \multirow{2}{*}{$-0.46[-0.94,0.02]$} & $22.68(8.95)$ & $25.46(7.91)$ & $-0.02[0.25,-0.30]$ & $-0.30[0.02,-0.63]$ \\
\hline & WLC & $22.87(9.12)$ & $23.02(10.37)$ & $-0.02[0.31,-0.35]$ & & NA & NA & NA & NA \\
\hline \multirow{2}{*}{ PHI } & UPHW & $6.20(2.24)$ & $6.43(1.91)$ & $-0.10[0.14,-0.35]$ & \multirow{2}{*}{$-0.30[-0.77,0.18]$} & $6.16(1.46)$ & $5.79(1.93)$ & $0.02[0.33,-0.30]$ & $0.18[0.46,-0.11]$ \\
\hline & WLC & $6.54(2.10)$ & $7.00(1.89)$ & $-0.22[0.08,-0.51]$ & & NA & NA & NA & NA \\
\hline \multirow{2}{*}{ SF-12 PH } & UPHW & $52.57(26.30)$ & $60.77(28.13)$ & $-0.30[0.10,-0.70]$ & \multirow{2}{*}{$-0.19[-0.67,0.28]$} & $61.61(32.67)$ & $63.13(25.57)$ & $-0.34[0.03,-0.71]$ & $-0.39[-0.10,-0.68]$ \\
\hline & WLC & $64.46(26.03)$ & $65.60(20.99)$ & $-0.04[0.31,-0.40]$ & & NA & NA & NA & NA \\
\hline \multirow{2}{*}{ SF-12 MH } & UPHW & $53.64(28.80)$ & $62.31(25.93)$ & $-0.29[0.08,-0.67]$ & \multirow{2}{*}{$0.03[-0.44,0.50]$} & $64.36(23.64)$ & $55.68(27.69)$ & $-0.36[0.08,-0.81]$ & $-0.07[0.25,-0.39]$ \\
\hline & WLC & $62.95(25.82)$ & $61.69(23.15)$ & $0.05[0.31,-0.22]$ & & NA & NA & NA & NA \\
\hline \multirow{2}{*}{ SF-12 GH } & UPHW & $53.11(24.07)$ & $61.54(24.20)$ & $-0.34[0.03,-0.71]$ & \multirow{2}{*}{$-0.10[-0.57,0.37]$} & $63.09(24.25)$ & $59.41(24.50)$ & $-0.41[0.01,-0.82]$ & $-0.26[0.03,-0.54]$ \\
\hline & WLC & $63.71(20.02)$ & $63.64(17.22)$ & $0.00[0.30,-0.29]$ & & NA & NA & NA & NA \\
\hline \multirow{2}{*}{ SSQ-N } & UPHW & $1.40(0.93)$ & $1.39(1.80)$ & $0.02[0.33,-0.30]$ & \multirow{2}{*}{$-0.32[-0.79,0.15]$} & $1.19(1.46)$ & $1.23(1.96)$ & $0.22[0.54,-0.10]$ & $0.18[0.66,-0.30]$ \\
\hline & WLC & $1.52(1.16)$ & $2.11(2.57)$ & $-0.49[-0.25,-0.73]$ & & NA & NA & NA & NA \\
\hline \multirow{2}{*}{ SSQ-S } & UPHW & $4.52(1.44)$ & $4.12(1.71)$ & $0.27[0.63,-0.09]$ & \multirow{2}{*}{$-0.52[-1.00,-0.04]$} & $3.97(2.13)$ & $3.69(2.47)$ & $0.37[0.74,-0.00]$ & $0.56[0.99,0.13]$ \\
\hline & WLC & $4.93(1.26)$ & $5.01(1.68)$ & $-0.06[0.22,-0.35]$ & & NA & NA & NA & NA \\
\hline
\end{tabular}




\section{3) Post module changes (ODSIS and OASIS)}

Inter-session means for anxiety (OASIS) and depression (ODSIS) are displayed in Figure 2. Repeated-measures ANOVA showed a significant time effect for both anxiety $\left(F_{(11,363)}=2.30, p=.01, \eta^{2} \mathrm{p}=.07 ; 1-\beta=.95\right)$ and depression $\left(F_{(11,363)}=2.35, p\right.$ $\left.=.008, \eta_{\mathrm{p}}^{2}=.07 ; 1-\beta=.95\right)$. Additionally, polynomial contrasts revealed evidence for a linear trend on both anxiety $\left(F_{(1,33)}=11.54, p=.002, \eta^{2} \mathrm{p}=.26 ; 1-\beta=.91\right)$ and depression $\left(F_{(1,33)}=13.62, p=.001, \eta^{2} \mathrm{p}=.29 ; 1-\beta=.95\right)$.

Figure 2. Inter-session means for anxiety (OASIS) and depression (ODSIS). The error bars represent the standard error for each $95 \%$ confidence interval.

\section{Post Module Changes}

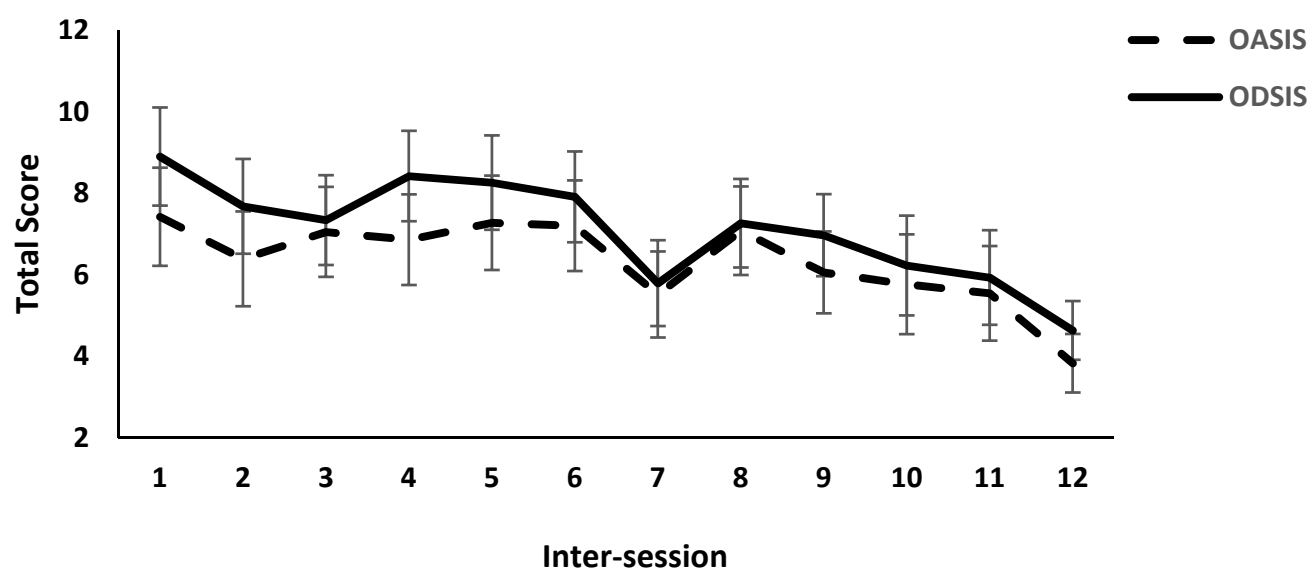

\section{4) Reliability of the clinical changes}

\section{Changes in depression (RCI for BDI)}

The RCI analyses indicated that there were significant differences between groups in the proportion of participants achieving a functional change on depression $\left(\chi^{2}(1)=6.02\right.$, $p=.01$ ), showing that the percentage of participants in UPHW group achieving functional changes $(78.8 \%)$ was larger than in WLC (43.8\%). Secondly, significant differences between group were also found in the reliability of change in depression $\left(\chi^{2}(2)=7.68, p\right.$ $=.02)$, revealing that the percentage of participants achieving reliable changes in depression was larger in UPHW (48.5\%) than in WLC $(18.8 \%)$. Furthermore, the percentage of participants deteriorated after the intervention was significantly larger in WLC $(25 \%)$ than in UPHW (3\%). Finally, Figure 3 shows that there were significant differences between UPHW and WLC in clinical change in depression $\left(\chi^{2}(3)=8.48, p=\right.$ $.03)$, where UPHW showed a higher number of participants recovered whereas WLC showed a higher number of no change and deteriorated participants.

\section{Changes in anxiety (RCI for BAI)}

Unlike depression, the RCI analyses indicated that the groups did not differ in the proportion of participants achieving a functional change on anxiety $\left(\chi^{2}(1)=.003, p=.95\right)$. Similarly, no significant differences between UPHW and WLC were found in the reliability of change in anxiety $\left(\chi^{2}(2)=.63, p=.73\right)$. Finally, Figure 3 shows that no significant differences between groups was found in clinical change in anxiety $\left(\chi^{2}(3)=\right.$ $1.39, p=.71)$. 
Figure 3. Reliable Change Index for primary outcomes measures. UPHW = Unified Protocol for homeless women; WLC $=$ Wait-list control period.
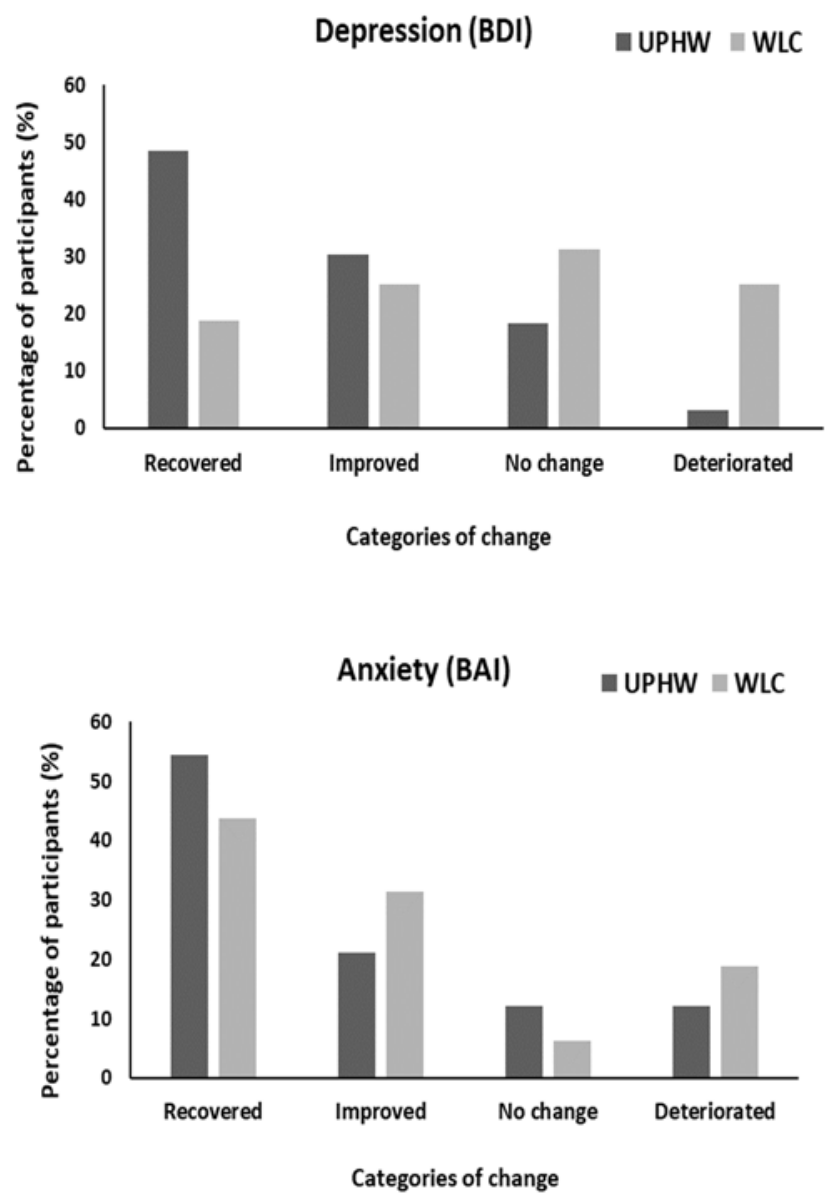

Discussion

Despite high rates of mental health problems and comorbidity in homeless people, there is a lack of evidence-based treatments adapted to specifically address the idiosyncratic characteristics of this population. Thus, the aim of this study was to evaluate the effectiveness of a group format adaptation of the Unified Protocol for homeless women. Transdiagnostic treatments in general, and the UP in particular, have supported this, as an effective alternative to traditional psychological treatments to address the difficulties arising from the interaction between the mental health problems and the lack of socioeconomic resources.

As predicted, the results of this study provide initial support for the use of UPHW adaptation for homeless women as an effective treatment to reduce psychological distress in this population. Homeless women receiving the UPHW demonstrated significant reductions of anxiety and depression symptoms when compared to WLC, as well as significant reductions in negative affect. Furthermore, effect size estimations suggest that the magnitude of treatment improvements were medium range ( $d$ negative affect $=0.4 ; d$ anxiety $=0.60$; and $d$ depression $=0.62$ symptoms). Interestingly, the reliability of the clinical changes showed significant differences between UPHW and WLC for depression; participants receiving the UPHW evidenced greater recovery (almost $50 \%$ of the cases) whereas 
participants in the WLC were more likely to show no change $(31 \%)$ or deterioration $(25 \%)$. However, no significant group differences were found in anxiety symptoms.

In terms of anxiety symptoms, the results found in our study are similar to those obtained in group format UP (Bullis et al., 2015; Osma et al., 2015) and individual UP (de Ornelas, et al., 2013; Farchione et al., 2012). We also found similar effect sizes in terms of depression (Osma et al., 2015) and negative affect (Farchione et al., 2012). In contrast with a previous study, where the effects of interventions for improving mental health outcomes in homeless people were limited (Baxter et al., 2019), our intervention for homeless women resulted in significant reductions in depression, anxiety and negative affect. These preliminary results suggest that the adapted UPHW may be an effective intervention for improving mental health in homeless people. Future studies should explore the transdiagnostic processes that could be responsible for these changes (e.g., negative and positive affect, emotional regulation, social support, etc.), analyzing the mediators and moderators of change in this intervention.

We did not find significant changes in positive affect following treatment. Some studies have reported improvements in positive affect following treatment with the UP (Farchione et al., 2012; Reinholt et al., 2017) whereas others have not (Ellard et al., 2010; Ito et al., 2016). The most recent version of the UP (Barlow, et al., 2018) places greater emphasis on reducing avoidance of positive emotions (e.g., "I do not deserve to be happy"). Nevertheless, the version of the UP used in our study (Barlow et al., 2011) is mainly focused on decreasing negative emotions such as anxiety and depression, and it gives little attention to the cultivation of positive emotions. Given the nature of emotions (i.e., positive and negative emotions do not exist on a bipolar continuum), the absence of changes in positive affect is not unusual. Although speaking of positive emotions and well-being in homeless people might seem inappropriate (e.g., "enough to survive to ask them to be happy"), it does seem to be an important factor to explain the mental health in this population (Panadero et al., 2015). For instance, Munoz et al. (2016) found that positive emotions contribute to health and wellbeing perceptions among homeless people, being an important mediator of quality of life. For this reason, future studies using UPHW should take into account positive affect and place a greater emphasis on increasing positive emotions while administering the primary treatment components.

Homeless women continued to show improvements in anxiety and depression symptoms three months following the end of intervention, lending preliminary support of its to the durability of the treatment effects over time. However, these improvements were no longer present at 6-month follow up. Most studies of the UP have found that reductions in anxiety and depression are maintained after 6 months (Barlow, et al., 2017; Farchione et al., 2012), with some studies even demonstrating maintenance of treatment gains over longer periods of time (Bullis et al., 2014). However, the clinical severity of the homeless population may be more significant than other clinical population, showing higher rates of anxiety and depression (Farchione et al., 2012). These differences may be due to the interaction between social exclusion and mental health problems, including the high rates of stressful life events to which these women are exposed every day, and the high rates of dual-diagnosis, among others. In fact, traumatic events and stress associated with experiences of homelessness may aggravate mental health problems (Duke \& Searby, 2019; Rodriguez-Moreno et al., 2020), which in turn influence homelessness chronification (Roca et al., 2019). Future studies should consider ways to improve longterm maintenance of treatment gains, such as introducing a "booster" session after the termination of treatment (e.g., 1 session after 2 and 4 month) to remind patients to practice 
treatment skills over time and help address new difficulties or stressors that they may encounter during the follow-up period.

We also predicted that the reductions in anxiety and depression symptoms would lead to improvements across a number of secondary outcome variables, such as wellbeing, health, and social support. Contrary to our expectations, we did not find significant changes in well-being, health variables and social support after the treatment. These results make sense since the UPHW version used in this study does not include specific modules aimed at improving these components, furthermore, these aspects can take time to change. However, all of these variables are especially relevant in homeless population. For this reason, future studies should consider introducing specific content, or additional treatment modules, to specifically address health care issues and strengthen social support networks in this population. For instance, some of the examples of adaptive emotion regulation in the original UP are related to enjoying activities with family or friends or doing activities that require physical mobility. Unfortunately, some of these basic elements of well-being may not always be present for homeless people. Social support networks, for example, may have dissolved or are very weak. Further, rates of physical conditions and disabilities tend to be high in this population. Thus, psychological interventions would constitute a key component of multifactorial community-based programs, together with other crucial structural factors such as housing or employment reintegration (Wickham, 2020). For instance, Housing First programs would benefit from the inclusion of psychological interventions like the UP (Tsemberis et al., 2004).

The results of the present study must be interpreted at the light of some methodological limitations. First, due to difficulties in recruiting participants across different treatment locations, we were unable to randomly assign participants to study condition. Thus, we cannot attribute with absolute certainty that the effects reported are due primarily to the intervention, as opposed to other additional variables, hence leading to potential risk of bias. Of course, randomized controlled trials are considered the "gold standard" for causal inference in health sciences, however, when interventions are applied to real-world community settings, such as the homeless shelters outside the idealized academic settings, its implementation is much more complex. Although many challenges were identified and addressed during the adaptation of the protocol, there were a number of implementation barriers encountered during the trial. The group format, together with the high dropout rates, made it difficult to form small groups (i.e., 5 participants). Given the risk that the group would eventually dissolve as treatment progressed (e.g., finishing the intervention with only 2 participants), we decided to delay the start of treatment until almost 10 participants began treatment at one time. The nomadic nature and the idiosyncratic characteristics of homeless population made continuity in the intervention more complex, as well as being able to locate participants for follow-up evaluations (e.g., some women changed the shelter or the city and it was not possible to contact them). Finally, homelessness generally leads to unstructured schedules, which made difficult the delivery of the intervention in terms of scheduling sessions and maintaining attendance. Other study limitations were as follows: 1) we did not use a structured interview to diagnose patients as it was considered too long for the population characteristics. Instead, we confirmed the referring diagnoses with the psychologist of the shelter; 2) the present study did not include an active treatment comparison, making it difficult to tease out the effect of common therapeutic factors (e.g., adherence, expectations, therapist attention, etc.); and 3) all participants were women, so future studies should include men in order to assess if there are gender differences in the response to the UPHW. 
These limitations notwithstanding, to our knowledge this is the first UP adaptation for homeless women in a group format, with data at 3-month and 6-month follow-up. The positive results achieved with this protocol have clinical implications, as protocols of this type could help reduce the burden on shelters, where there is usually only one psychologist on staff. Furthermore, the group format of the UPHW used in this trial fit especially well with the structure and needs of homeless shelters (i.e., cost-effective interventions). As observed in de Ornelas and colleagues' study (2013), participants felt like part of a group with the same characteristics, which encouraged them to share information and improve their social support networks. While previous treatments for homelessness have focused more on structural factors (Hwang \& Burns, 2014), we have adapted a purely psychological treatment to directly address the psychological needs of the population. Finally, we believe our study makes an important contribution to the current literature on transdiagnostic protocols in socially excluded populations (SauerZavala et al., 2019).

\section{Conclusions}

The preliminary results of our study suggest that the UPHW adaptation for homeless women can be a feasible and effective treatment for depression, anxiety and negative affect in this population. Further research is warranted to replicate our findings and to provide additional evidence of its efficacy and effectiveness in other groups of people who experience social exclusion situations. Furthermore, these data encourage us to perform clinical trials to establish the effectiveness of the UP in group format not only in clinical populations, but also in social settings.

\section{References}

Amore, K., Baker, M., \& Howden-Chapman, P. (2011). The ETHOS Definition and Classification of Homelessness: An Analysis. European Journal of Homelessness, $5(2)$.

Australian Bureau of Statistics. (2016). Mental health and experiences of homelessness, Australia, 2014 (No 4329.0.00.005). ACT.

Barlow, D. H., Farchione, T. J., Bullis, J. R., Gallagher, M. W., Murray-Latin, H., SauerZavala, S., Bentley, K. H., Thompson-Hollands, J., Conklin, L. R., Boswell, J. F., Ametaj, A., Carl, J. R., Boettcher, H. T., \& Cassiello-Robbins, C. (2017). The unified protocol for transdiagnostic treatment of Emotional Disorders compared with diagnosis-specific protocols for anxiety disorders: A randomized clinical trial. JAMA Psychiatry, 74(9), 875-884. https://doi.org/10.1001/jamapsychiatry.2017.2164

Barlow, D. H., Farchione, T. J., Sauer-Zavala, S., Murray Latin, H., Ellard, K. K., Bullis, J. R., Bentley, K. H., Boettcher, H. T., \& Cassiello-Robbins, C. (2018). Unified protocol for transdiagnostic treatment of emotional disorders. Second Edition. Oxford University Press.

Barlow, D. H., Farchione, T. J., Fairholme, C. P., Ellard, K. K., Boisseau, C. L., Allen, L. B., \& Ehrenreich-May, J. (2011). The unified protocol for transdiagnostic treatment of emotional disorders: Therapist guide. Oxford University Press.

Barlow, D. H., Sauer-Zavala, S., Carl, J. R., Bullis, J. R., \& Ellard, K. K. (2014). The nature, diagnosis, and treatment of neuroticism: Back to the future. Clinical Psychological Science, 2(3), 344-365. https://doi.org/10.1177/2167702613505532 
Baxter, A. J., Tweed, E. J., Katikireddi, S. V., \& Thomson, H. (2019). Effects of Housing First approaches on health and well-being of adults who are homeless or at risk of homelessness: Systematic review and meta-analysis of randomised controlled trials. Journal of Epidemiology and Community Health, 73(5), 379-387. https://doi.org/10.1136/jech-2018-210981

Bullis, J. R., Fortune, M. R., Farchione, T. J., \& Barlow, D. H. (2014). A preliminary investigation of the long-term outcome of the Unified Protocol for Transdiagnostic Treatment of Emotional Disorders. Comprehensive Psychiatry, 55(8), 1920-1927. https://doi.org/10.1016/j.comppsych.2014.07.016

Bullis, J. R., Sauer-Zavala, S., Bentley, K. H., Thompson-Hollands, J., Carl, J. R., \& Barlow, D. H. (2015). The unified protocol for transdiagnostic treatment of emotional disorders: Preliminary exploration of effectiveness for group delivery. Behavior Modification, 39(2), 295-321. https://doi.org/10.1177/0145445514553094

Chambers, C., Chiu, S., Scott, A. N., Tolomiczenko, G., Redelmeier, D. A., Levinson, W., \& Hwang, S. W. (2014). Factors associated with poor mental health status among homeless women with and without dependent children. Community Mental Health Journal, 50(5), 553-559. https://doi.org/10.1007/s10597-013-9605-7

Coldwell, C. M., \& Bender, W. S. (2007). The Effectiveness of Assertive Community Treatment for Homeless Populations With Severe Mental Illness: A Meta-Analysis. American Journal of Psychiatry, 164(3), 393-399. https://doi.org/10.1176/ajp.2007.164.3.393

de Ornelas, A., Braga, A., Nunes, C., Nardi, A., \& Cardoso, A. (2013). Transdiagnostic treatment using a unified protocol: application for patients with a range of comorbid mood and anxiety disorders. Trends in Psichiatry and Psychotherapy, 35(2), 134140. https://doi.org/https://doi.org/10.1590/S2237-60892013000200007

Duke, A., \& Searby, A. (2019). Mental ill health in homeless women: A review. Issues in Mental Health Nursing, 40(7), 605-612. https://doi.org/10.1080/01612840.2019.1565875

Ellard, K. K., Fairholme, C. P., Boisseau, C. L., Farchione, T. J., \& Barlow, D. H. (2010). Unified protocol for the transdiagnostic treatment of emotional disorders: Protocol development and initial outcome data. Cognitive and Behavioral Practice, 17(1), 88-101. https://doi.org/10.1016/j.cbpra.2009.06.002

Farchione, T. J., Fairholme, C. P., Ellard, K. K., Boisseau, C. L., Thompson-Hollands, J., Carl, J. R., Gallagher, M. W., \& Barlow, D. H. (2012). Unified protocol for transdiagnostic treatment of emotional disorders: A randomized controlled trial. Behavior Therapy, 43(3), 666-678. https://doi.org/10.1016/j.beth.2012.01.001

Fazel, S., Geddes, J. R., \& Kushel, M. (2014). The health of homeless people in highincome countries: Descriptive epidemiology, health consequences, and clinical and policy recommendations. The Lancet, 384(9953), 1529-1540. https://doi.org/10.1016/S0140-6736(14)61132-6

Fazel, S., Khosla, V., Doll, H., \& Geddes, J. (2008). The prevalence of mental disorders among the homeless in Western countries: Systematic review and meta-regression analysis. $\quad P L o S \quad$ Medicine, 1670-1681. 
https://doi.org/10.1371/journal.pmed.0050225

Fransham, M., \& Dorling, D. (2018). Homelessness and public health. In BMJ (Online) (Vol. 360). BMJ. https://doi.org/10.1136/bmj.k214

Hossain, M. M., Sultana, A., Tasnim, S., Fan, Q., Ma, P., McKyer, E. L. J., \& Purohit, N. (2020). Prevalence of mental disorders among people who are homeless: An umbrella review. International Journal of Social Psychiatry, 66(6), 528-541. https://doi.org/10.1177/0020764020924689

Hwang, S. W., \& Burns, T. (2014). Health interventions for people who are homeless. The Lancet, 384(9953), 1541-1547. https://doi.org/10.1016/S0140-6736(14)611338

Ito, M., Horikoshi, M., Kato, N., Oe, Y., Fujisato, H., Nakajima, S., Kanie, A., Miyamae, M., Takebayashi, Y., Horita, R., Usuki, M., Nakagawa, A., \& Ono, Y. (2016). Transdiagnostic and transcultural: Pilot study of unified protocol for depressive and anxiety disorders in Japan. Behavior Therapy, 47(3), 416-430. https://doi.org/10.1016/j.beth.2016.02.005

Jacobson, N. S., \& Truax, P. (1991). Clinical significance: A statistical approach to defining meaningful change in psychotherapy research. Journal of Consulting and Clinical Psychology, 59(1), 12-19.

Jiga, K., Kaunhoven, R. J., \& Dorjee, D. (2019). Feasibility and efficacy of an adapted Mindfulness-Based Intervention (MBI) in areas of socioeconomic deprivation (SED). Mindfulness, 10(2), 325-338. https://doi.org/10.1007/s12671-018-0977-1

Lako, D. A. M., De Vet, R., Beijersbergen, M. D., Herman, D. B., Van Hemert, A. M., \& Wolf, J. R. (2013). The effectiveness of critical time intervention for abused women and homeless people leaving Dutch shelters: Study protocol of two randomised controlled trials. BMC Public Health, 13(1), 1-12. https://doi.org/10.1186/14712458-13-555

Llerena, K., Gabrielian, S., \& Green, M. F. (2018). Clinical and cognitive correlates of unsheltered status in homeless persons with psychotic disorders. Schizophrenia Research, 197, 421-427. https://doi.org/10.1016/j.schres.2018.02.023

Lund, C., Breen, A., Flisher, A. J., Kakuma, R., Corrigall, J., Joska, J. A., Swartz, L., \& Patel, V. (2010). Poverty and common mental disorders in low and middle income countries: A systematic review. Social Science \& Medicine, 71(3), 517-528. https://doi.org/10.1016/J.SOCSCIMED.2010.04.027

Martens, W. (2001). A review of physical and mental health in homeless persons. Public Health Reviews, 29(1), 13-33.

Mayock, P., \& Sheridan, S. (2012). Women's “Journeys” to homelessness: Key findings from a biographical study of homeless women in Ireland. School of Social Work and Social Policy and Children's Research Centre, Trinity College Dublin.

Mayock, P., \& Bretherton, J. (2016). Women's homelessness in Europe. Palgrave Macmillan UK.

McHugh, R. K., Murray, H. W., \& Barlow, D. H. (2009). Balancing fidelity and adaptation in the dissemination of empirically-supported treatments: The promise of transdiagnostic interventions. Behaviour Research and Therapy, 47(11), 946-953. 
https://doi.org/10.1016/j.brat.2009.07.005

Moher, D., Hopewell, S., Schulz, K. F., Montori, V., Gøtzsche, P. C., Devereaux, P. J., Elbourne, D., Egger, M., \& Altman, D. G. (2012). CONSORT 2010 explanation and elaboration: Updated guidelines for reporting parallel group randomised trials. International Journal of Surgery, 10(1), 28-55. https://doi.org/10.1016/j.ijsu.2011.10.001

Muñoz, M., Crespo, M., \& Pérez-Santos, E. (2005). Homelessness effects on men's and women's health. International Journal of Mental Health, 34(2), 47-61. https://doi.org/10.1080/00207411.2005.11043400

Munoz, R. T., Hellman, C. M., Buster, B., Robbins, A., Carroll, C., Kabbani, M., Cassody, L., Brahm, N., \& Fox, M. D. (2016). Life satisfaction, hope, and positive emotions as antecedents of health related quality of life among homeless individuals. International Journal of Applied Positive Psychology, 1(1-3), 69-89. https://doi.org/10.1007/s41042-017-0005-z

National Institute of Mental Health. (2009). Anxiety disorders (NIH Publication No. 093879). http://www.nimh.nih.gov/health/publications/anxietydisorders/nimhanxiety.pdf

National Institute of Mental Health. (2017). Statistics. https://www.nimh.nih.gov/health/statistics/

National Institute of Statistics. (2018). Encuesta sobre centros y servicios de atención a personas sin hogar.

Newman, D. A. (2014). Missing data: Five practical guidelines. Organizational Research Methods, 17(4), 372-411. https://doi.org/10.1177/1094428114548590

Nilsson, S. F., Nordentoft, M., \& Hjorthøj, C. (2019). Individual-level predictors for becoming homeless and exiting homelessness: a systematic review and metaanalysis. Journal of Urban Health, 96(5), 741-750. https://doi.org/10.1007/s11524019-00377-x

Osma, J., Delgado, E. C., \& Garcia-Palacios, A. (2015). The unified protocol for transdiagnostic treatment of emotional disorders in group format in a spanish public mental health setting. Behavioral Psychology, 23(3), 447-466.

Osma, J., Suso-Ribera, C., García-Palacios, A., Crespo-Delgado, E., Robert-Flor, C., Sánchez-Guerrero, A., Ferreres-Galan, V., Pérez-Ayerra, L., Malea-Fernández, A., \& Torres-Alfosea, M. Á. (2018). Efficacy of the unified protocol for the treatment of emotional disorders in the Spanish public mental health system using a group format: Study protocol for a multicenter, randomized, non-inferiority controlled trial. Health and Quality of Life Outcomes, 16(1), 1-10. https://doi.org/10.1186/s12955-018-0866-2

Panadero, S., Guillén, A. I., \& Vázquez, J. J. (2015). Happiness on the street: Overall happiness among homeless people in Madrid (Spain). American Journal of Orthopsychiatry, 85(4), 324-330. https://doi.org/10.1037/ort0000080

Reinholt, N., Aharoni, R., Winding, C., Rosenberg, N., Rosenbaum, B., \& Arnfred, S. (2017). Transdiagnostic group CBT for anxiety disorders: The unified protocol in mental health services. Cognitive Behaviour Therapy, 46(1), 29-43. 
https://doi.org/10.1080/16506073.2016.1227360

Rew, L., Powell, T., Brown, A., Becker, H., \& Slesnick, N. (2017). An intervention to enhance psychological capital and health outcomes in homeless female youths. Western Journal of Nursing Research, 39(3), 356-373. https://doi.org/10.1177/0193945916658861

Roca, P., Panadero, S., Rodríguez-Moreno, S., Martín, R. M., \& Vázquez, J. J. (2019). The revolving door to homelessness. The influence of health, alcohol consumption and stressful life events on the number of episodes of homelessness. Annals of Psychology, 35(2), 175-180. https://doi.org/10.6018/ANALESPS.35.2.297741

Rodriguez-Moreno, S., Panadero, S., \& Vázquez, J. J. (2020). Risk of mental ill-health among homeless women in Madrid (Spain). Archives of Women's Mental Health, 23, 657-664. https://doi.org/10.1007/s00737-020-01036-w

Sakiris, N., \& Berle, D. (2019). A systematic review and meta-analysis of the Unified Protocol as a transdiagnostic emotion regulation based intervention. Clinical Psychology Review, 72, 101751. https://doi.org/10.1016/j.cpr.2019.101751

Santa Maria, D., Cuccaro, P., Bender, K., Cron, S., Fine, M., \& Sibinga, E. (2020). Feasibility of a Mindfulness-Based Intervention with sheltered youth experiencing homelessness. Journal of Child and Family Studies, 29(1), 261-272. https://doi.org/10.1007/s10826-019-01583-6

Sauer-Zavala, S., Ametaj, A. A., Wilner, J. G., Bentley, K. H., Marquez, S., Patrick, K. A., Starks, B., Shtasel, D., \& Marques, L. (2019). Evaluating transdiagnostic, evidence-based mental health care in a safety-net setting serving homeless individuals. Psychotherapy, 56(1), 100-114. https://doi.org/10.1037/pst0000187

Schminder, E., Ziegler, M., Danay, E., Beyer, L., \& Bühner, M. (2010). Is it really robust? Reinvestigating the robustness of ANOVA against violations of the normal distribution. European Research Journal of Methods for the Behavioral and Social Sciences, 6, 147-151.

Schreiter, S., Bermpohl, F., Krausz, M., Leucht, S., Rössler, W., Schouler-Ocak, M., \& Gutwinski, S. (2017). The prevalence of mental illness in homeless people in Germany - A systematic review and meta-analysis. Deutsches Arzteblatt International, 114(40), 665-672. https://doi.org/10.3238/arztebl.2017.0665

Speirs, V., Johnson, M., \& Jirojwong, S. (2013). A systematic review of interventions for homeless women. Journal of Clinical Nursing, 22(7-8), 1080-1093. https://doi.org/10.1111/jocn.12056

Tsemberis, S., Gulcur, L., \& Nakae, M. (2004). Housing First, consumer choice, and harm reduction for homeless individuals with a dual diagnosis. American Journal of Public Health, 94(4), 651-656. https://doi.org/10.2105/AJPH.94.4.651

U.S. Department of Housing and Urban Development. (2015). The 2015 Annual Homeless Assessment Report (AHAR) to Congress. https://www.hudexchange.info/onecpd/assets/File/2015-AHAR-Part-2.pdf. rt-2.pdf

Urbanoski, K., Veldhuizen, S., Krausz, M., Schutz, C., Somers, J. M., Kirst, M., Fleury, M.-J., Stergiopoulos, V., Patterson, M., Strehlau, V., \& Goering, P. (2018). Effects of comorbid substance use disorders on outcomes in a Housing First intervention for 
homeless people with mental illness. Addiction, 113(1), 137-145. https://doi.org/10.1111/add.13928

Vázquez, C., \& Muñoz, M. (2001). Homelessness, mental health, and stressful life events: The Madrid experience. International Journal of Mental Health, 30(3), 6-25. https://doi.org/10.1080/00207411.2001.11449522

Watson, J., Crawley, J., \& Kane, D. (2016). Social exclusion, health and hidden homelessness. Public Health, 139, 96-102. https://doi.org/10.1016/j.puhe.2016.05.017

Welch-Lazoritz, M. L., Whitbeck, L. B., \& Armenta, B. E. (2015). Characteristics of mothers caring for children during episodes of homelessness. Community Mental Health Journal, 51(8), 913-920. https://doi.org/10.1007/s10597-014-9794-8

Wickham, S. (2020). Effective interventions for homeless populations: The evidence remains unclear. The Lancet Public Health, 5(6), e304-e305. https://doi.org/10.1016/S2468-2667(20)30120-1

Youn, S. J., Sauer-Zavala, S., Patrick, K. A., Ahles, E. M., Aguilar Silvan, Y., Greig, A., Marques, L., \& Shtasel, D. L. (2019). Barriers and facilitators to implementing a short-term transdiagnostic mental health treatment for homeless persons. The Journal of Nervous and Mental Disease, 207(7), 585-594. https://doi.org/10.1097/NMD.0000000000001010 


\section{ANNEX:}

\section{SUPPLEMENTARY}

MATERIALS 


\section{Method}

\section{Treatment}

Supplementary Table 1. Treatment content split by session.

\begin{tabular}{|c|c|}
\hline Session number & Content \\
\hline Session 1 & $\begin{array}{l}\text { Psychoeducation. Understanding emotions. Introduction to } \\
\text { the motivation to change }\end{array}$ \\
\hline Session 2 & Motivation and commitment to change. Objectives and goals \\
\hline Session 3 & $\begin{array}{l}\text { Three components model of emotional experiences. What do } \\
\text { I think? what do I feel? how do I act? }\end{array}$ \\
\hline Session 4 & $\begin{array}{l}\text { Recognizing and tracking emotional responses. ARC model } \\
\text { of emotional experiences }\end{array}$ \\
\hline Session 5 & $\begin{array}{l}\text { Emotion awareness training. Non-judgmental present- } \\
\text { focused emotion awareness exercises Mindfulness }\end{array}$ \\
\hline Session 6 & $\begin{array}{l}\text { Understanding the thought: Evaluation and cognitive } \\
\text { reevaluation. Cognitive flexibility }\end{array}$ \\
\hline Session 7 & $\begin{array}{l}\text { Understanding and managing our feelings: Awareness and } \\
\text { tolerance to physical sensations }\end{array}$ \\
\hline Session 8 & Emotion-driven behaviors (EDBs) \\
\hline Session 9 & Emotional Avoidance \\
\hline Session 10 & $\begin{array}{l}\text { Interoceptive and situational emotion exposure. Imaginal } \\
\text { exposure }\end{array}$ \\
\hline Session 11 & Achievement Recognition and looking to the future \\
\hline Session 12 & Relapse prevention and treatment termination \\
\hline
\end{tabular}

EDBs: Emotion-driven behaviors

\section{Therapist and treatment integrity}

The therapist (SRM) and the co-therapist were two $\mathrm{PhD}$ candidates in clinical psychology with extensive experience working with homeless individuals in clinical practice. Both the therapist and the co-therapist were trained in the UPHW protocol by the treatment developers, and both were directly involved in the adaptation of the treatment protocol. Program adherence was supported by periodic meetings with the senior authors of the study. Furthermore, treatment adherence was assessed by the therapist and co-therapist after each session using a standardized questionnaire assessing degree of adherence to the goals, contents and activities of each session, as well as an open-ended question about areas for improvement (Marín et al., unpublished).

Therapists contacted participants before treatment sessions and also after any missed sessions to explore reasons for non-attendance. Previous studies have shown that dropout rates are very high in homeless population (Coldwell \& Bender, 2007; Rew et al., 2017), probably due to the nomadic nature of the homeless. In this study, treatment completion was high, with only $28.3 \%$ discontinuing treatment in the UPHW. This may be due to the high satisfaction levels $(M=8.97, S D=1.45$ in a single-item measuring satisfaction from 0 to 10$)$ and the perceived usefulness of the treatment $(M=9.10$, $S D=1.42$; in a single-item measuring usefulness from 0 to 10 ) in the full sample (including the drop-out cases). that have been reported for the UPHW in this population (Marin et 
al., unpublished). Reasons for treatment discontinuation included health problems, hospitalizations, incompatibility with medical appointments, and alcohol and other substance problems.

\section{Assessment}

\section{Primary outcomes}

Beck Anxiety Inventory (BAI) (Beck \& Steer, 1993). The BAI is a 21-item selfreport measure designed to assess anxiety severity, with a maximum of 63 points. Each item has a 4-point severity scale (e.g., not at all, mildly, moderately, and severely), addressing symptoms experienced during the past week. The internal consistency of the Spanish version has been found to range from .85 to .94 (Magán et al., 2008). In the current study, the internal consistency of the BAI was $\alpha=.89$.

Beck Depression Inventory-II (BDI-II) (Beck et al., 1990). The BDI-II is a 21item self-report measure designed to assess depression severity. Each item is rated from 0 to 3 with a total score of a maximum of 63 points. The Spanish version has shown good internal consistency $(\alpha=0.86)$ (Sanz et al., 2003). In the current study, the internal consistency of the BDI-II was $\alpha=.90$.

\section{Secondary outcomes}

Positive and Negative Affect Scale (PANAS) (Watson et al., 1988). The PANAS is a 20-item self-report measure designed to assess negative and positive affect in the last week. Each item has a 5-point severity scale (e.g., not at all, mildly, moderately, quite a lot, and severely) with a total score from 10 to 50 in each factor. The internal consistency of the Spanish version was $\alpha=.92$ for positive affect subscale and $\alpha=.88$ for negative affect subscale (López-Gómez et al., 2015). In the current study, Cronbach's alpha was $\alpha$ $=.89$ for the positive affect subscale, and $\alpha=.84$ for the negative affect subscale.

Pemberton Happiness Index (PHI) (Hervás \& Vázquez, 2013). The PHI is a 21item measure designed to assess integrative well-being using a scale from 0 (fully disagree) to 10 (fully agree) in the first 11 items and dichotomous answers (yes/no) for the last 10 items. It includes two subscales (remember well-being and experienced wellbeing) and a total score. Remember well-being subscale assesses happiness and satisfaction level based upon people' memory and judgment of their lives, while experienced well-being subscale assesses momentary affective states in real time. The total mean score ranges from 0 to 10 and the Cronbach's alpha of Spanish version was $\alpha$ $=.84$. In the current study, the internal consistency of the PHI was $\alpha=.85$.

Short Form Health Survey (SF-12) (Ware et al., 1996). SF-12 is a generic health status instrument with 12 items, and eight subscales (physical functioning, role physical, bodily pain, general health, vitality, social functioning, role emotional and mental health). These eight subscales can be combined into two scores for physical health (PH) and mental health $(\mathrm{MH})$, and a total score, ranged from 0 to 100 , where higher scores reflect better self-reported health. The internal consistency of the Spanish version was $\alpha=.85$ for $\mathrm{PH}$ and $\alpha=.78$ for $\mathrm{MH}$ (Vilagut et al., 2008). In the current study, Cronbach's alpha was $\alpha=.79$ for the PH subscale, and $\alpha=.75$ for the MH subscale.

Social Support Questionnaire (SSQ6) (Sarason et al., 1987): The SSQ is a 6 item measure of social support. It includes two scales: number and satisfaction with social support (on a scale ranging from 1 to 9). The Spanish version has shown good internal consistency for both dimensions ( $\alpha=0.90$ and $\alpha=0.93$, respectively) (Martínez-López et al., 2014). In the current study, the internal consistency of the SSQ6 was $\alpha=.89$. 
Overall Anxiety Severity and Impairment Scale (OASIS) (Norman et al., 2006) and Overall Depression Severity and Impairment Scale (ODSIS) (Bentley et al., 2014). The OASIS and ODSIS are two self-report questionnaire designed to evaluate the severity and functional impairment associated with anxiety and depression, respectively. The scales consist of five items with a 5-point Likert scale ranging from 0 (I didn't feel anxious/depressed) to 4 (Constant anxiety/depressed); total scores range from 0 to 20. Cronbach's alpha of the OASIS Spanish version was 0.86 (González-Robles et al., 2018) and $\alpha=.93$ for the ODSIS Spanish version (Mira et al., 2019). In the present study, the Cronbach's alpha for the OASIS was $\alpha=.86$ and for the ODSIS was $\alpha=.89$.

\section{Data analysis}

Student $t$ and chi-square test were used to analyze baseline differences between groups. Following CONSORT guidelines (Moher et al., 2012), data imputation was performed following Newman's suggestions (2014), using Maximum Likelihood estimation (ML) via Expectation Maximization imputation (EM). The treatment of missing data was conducted following Hair and colleagues (2014) recommendations. Firstly, construct-level missing data were found, with a $16.2 \%$ of overall pre-post missing values in both groups and $22.5 \%$ of missing values along the four temporal moments in UPHW group. Secondly, Little's MCAR test, which was used to evaluate the random pattern of missing data, showed that the missing data were completely at random $\left(\chi^{2}(248)\right.$ $=168.60, p>.05)$, and thus suitable for imputation. Thirdly, no significant differences between completers and dropped out cases were found in age $\left(t_{(44)}=-0.84, p=.41\right)$, nationality $\left(\chi_{(2)}^{2}=2.34, p=.31\right)$, education $\left(\chi_{(5)}^{2}=3.70, p=.60\right)$, marital status $\left(\chi^{2}(1)=\right.$ $0.93, p=.34)$, and employment $\left(\chi^{2}(1)=1.13, p=.29\right)$. Furthermore, no significant differences between groups were found in the age of arrival to a homeless situation $\left(t_{(42)}\right.$ $=-0.14, p=.89)$, in the total time in a homeless situation $\left(t_{(41)}=-1.03, p=.31\right)$, and in the number of times in a homeless situation $\left(t_{(43)}=-0.81, p=.42\right)$. Finally, after the ML imputation, sensitivity analysis was conducted comparing the results of the completers with the estimated data in the main outcomes (i.e., BDI and BAI), reaching the same conclusions with both data sets, indicating that ML estimation would not lead to biased estimations.

The data analysis plan was conducted with SPSS v. 25 following four successive steps. Firstly, analyses of covariance (ANCOVA) were carried out to examine the pre to post intervention effects in primary and secondary outcomes, using condition as the between-subjects factor (i.e., UPHW vs WLC) and baseline scores as covariates (i.e., pre scores). The use of ANCOVAs while controlling baseline scores has been recommended by several authors as a more powerful tool in pretest-posttest designs (Weinfurt, 2000). Secondly, in order to test whether the post-intervention changes remain stable over time, repeated measure ANOVAs were computed for the follow ups (i.e., post, 3-months and 6-months follow up). Thirdly, repeated measure ANOVA was performed to test postmodule changes in depression (ODSIS) and anxiety (OASIS) in the UPHW group, using polynomial contrasts to test linear trends in the outcomes (i.e., 12 inter session assessment). For all the analyses: a) basic assumptions were tested, correcting the degrees of freedom when sphericity assumption was violated; b) Partial Eta square $\left(\eta^{2} \mathrm{p}\right)$ and Cohen's d effect sizes and its corresponding 95\% Confidence Intervals (CI) were calculated based on Botella \& Sánchez-Meca suggestions (2015); power analysis (1- $\beta$ ) was also computed for each ANOVA effect; and d) pairwise Bonferroni-corrected comparisons were used for post-hoc analysis. 
Finally, in order to improve individual-level analysis and the detection of potential adverse effects of the intervention, the Reliable Change Index was computed using the Jacobson and Truax's index (RCI; Jacobson \& Truax, 1991) for the main outcome measures (i.e., BDI and BAI) in the completer's database. Firstly, we established the cutoff points for each post-intervention outcome (i.e., under cut-off = no change; above cutoff = functional change). Secondly, the RCI was computed, where an RCI lower than 1.96 indicates an improvement, an RCI between -1.96 and 1.96 indicates no changes and RCI greater than 1.96 indicates a deterioration. Finally, in order to determine the type of clinical change, participants were classified into four categories using their cut-off and RCI scores: a) No change: when the post-intervention score does not reach the functional cut-off and the change is no reliable, b) Recovered: when post-intervention scores is located within the range of the functional distribution and the change is reliable; c) Improved: when the change is reliable but post-intervention scores does not reach the functional level; and d) Deteriorated: when the post-intervention score does not reach the functional cut-off and the post-intervention score is worse than pre-intervention score.

\section{References}

Beck, A., \& Steer, R. (1993). Beck anxiety inventory manual. Psychological Corporation.

Beck, A., Steer, R., \& Brown, G. (1990). Manual for the Beck Depression Inventory-II. Psychological Corporation.

Bentley, K. H., Gallagher, M. W., Carl, J. R., \& Barlow, D. H. (2014). Development and validation of the Overall Depression Severity and Impairment Scale. Psychological Assessment, 26(3), 815-830. https://doi.org/10.1037/a0036216

Botella, J., \& Sánchez-Meca, J. (2015). Meta-análisis en Ciencias Sociales y de la Salud [meta-analysis in social and health sciences]. Sintesis.

Coldwell, C. M., \& Bender, W. S. (2007). The Effectiveness of Assertive Community Treatment for Homeless Populations With Severe Mental Illness: A Meta-Analysis. American Journal of Psychiatry, 164(3), 393-399. https://doi.org/10.1176/ajp.2007.164.3.393

González-Robles, A., Mira, A., Miguel, C., Molinari, G., Díaz-García Id, A., GarcíaPalacios, A., Bretón-López, J. M., Quero, S., Baños, R. M., \& Botella, C. (2018). A brief online transdiagnostic measure: Psychometric properties of the Overall Anxiety Severity and Impairment Scale (OASIS) among Spanish patients with emotional disorders. PLoS One, 13(11), e206516. https://doi.org/10.1371/journal.pone.0206516

Hair, J. F., Black, W. C., Babin, B. J., \& Anderson, R. E. (2014). Multivariate Data Analysis, 7th edition. Pearson-Prentice Hall International Edition.

Hervás, G., \& Vázquez, C. (2013). Construction and validation of a measure of integrative well-being in seven languages: The Pemberton Happiness Index. Health and Quality of Life Outcomes, 11(1), 1-13. https://doi.org/10.1186/1477-7525-11-66

Jacobson, N. S., \& Truax, P. (1991). Clinical significance: A statistical approach to defining meaningful change in psychotherapy research. Journal of Consulting and Clinical Psychology, 59(1), 12-19.

López-Gómez, I., Hervás, G., \& Vázquez, C. (2015). Adaptación de las "Escalas de afecto positivo y negativo" (PANAS) en una muestra general española. Psicología Conductual, 23(3), 529-548. 
Magán, I., Sanz, J., \& Garcia-Vera, M. P. (2008). Psychometric properties of a Spanish version of the Beck Anxiety Inventory (BAI) in general population. The Spanish Journal of Psychology, 11(2), 626-640. https://doi.org/https://doi.org/10.1017/S1138741600004637

Martínez-López, Z., Páramo, M. F. ., Guisande, M. A., Tinajero, C., da Silva, L., \& Rodríguez, M. S. (2014). Social support in Spanish first year university students: Psychometrics properties of the Social Support Questionnaire-Short Form and the Social Provisions Scale. Revista Latinoamericana de Psicología, 46(2), 102-110. https://doi.org/10.1016/S0120-0534(14)70013-5

Mira, A., González-Robles, A., Molinari, G., Miguel, C., Díaz-García, A., Bretón-López, J., García-Palacios, A., Quero, S., Baños, R., \& Botella, C. (2019). Capturing the severity and impairment associated with depression: The Overall Depression Severity and Impairment Scale (ODSIS) validation in a Spanish clinical sample. Frontiers in Psychiatry, 10, 180. https://doi.org/10.3389/fpsyt.2019.00180

Moher, D., Hopewell, S., Schulz, K. F., Montori, V., Gøtzsche, P. C., Devereaux, P. J., Elbourne, D., Egger, M., \& Altman, D. G. (2012). CONSORT 2010 explanation and elaboration: Updated guidelines for reporting parallel group randomised trials. International Journal of Surgery, 10(1), 28-55. https://doi.org/10.1016/j.ijsu.2011.10.001

Newman, D. A. (2014). Missing data: Five practical guidelines. Organizational Research Methods, 17(4), 372-411. https://doi.org/10.1177/1094428114548590

Norman, S. B., Cissell, S. H., Means-Christensen, A. J., \& Stein, M. B. (2006). Development and validation of an Overall Anxiety Severity and Impairment Scale (OASIS). Depression and Anxiety, 23(4), 245-249. https://doi.org/10.1002/da.20182

Rew, L., Powell, T., Brown, A., Becker, H., \& Slesnick, N. (2017). An intervention to enhance psychological capital and health outcomes in homeless female youths. Western Journal of Nursing Research, 39(3), 356-373. https://doi.org/10.1177/0193945916658861

Sanz, J., Navarro, M., \& Vázquez, C. (2003). Adaptación española del Inventario para la Depresión de Beck-II (BDI-II): 1. Propiedades psicométricas en estudiantes universitarios. Análisis y Modificación de Conducta, 29, 239-288.

Sarason, I. G., Sarason, B. R., Shearin, E. N., \& Pierce, G. R. (1987). A brief measure of social support: Practical and theoretical implications. Journal of Social and Personal Relationships, 4(4), 497-510. https://doi.org/10.1177/0265407587044007

Vilagut, G., Valderas, J., Ferrer, M., Garin, O., López-García, E., \& Alonso, J. (2008). Interpretación de los cuestionarios de salud SF-36 y SF-12 en España: componentes físico y mental. Medicina Clínica, 130(19), 726-735. https://doi.org/10.1157/13121076

Ware, J. E., Kosinski, M., \& Keller, S. D. (1996). A 12-Item Short-Form Health Survey: Construction of scales and preliminary tests of reliability and validity. Medical Care, 220-233.

Watson, D., Clark, L. A., \& Tellegen, A. (1988). Development and validation of brief measures of positive and negative affect: The PANAS scales. Journal of Personality and Social Psychology, 54(6), 1063-1070. 
Weinfurt, K. P. (2000). Reading and understanding MORE multivariate statistics. In In L. G. Grimm \& P. R. Yarnold (Eds.) (American P, pp. 317-361). 